\title{
Nurses' roles in the management of chronic inflammatory arthritis: a systematic review
}

\author{
Lion Vivienne ${ }^{1} \cdot$ Schirmer Michael ${ }^{1}$ (1)
}

Received: 11 June 2018 / Accepted: 14 August 2018 / Published online: 20 August 2018

(c) The Author(s) 2018

\begin{abstract}
In 2011 EULAR first published recommendations for the potential role of nurses in the management of patients with rheumatic diseases. To perform a literature update for the role of nurses in the management of chronic inflammatory arthritis (CIA) from 2010 to 2018. A systematic literature review (SLR) was performed according to the PRISMA guidelines, in accordance with the search strategies and eligibility criteria of the EULAR taskforce. The eligibility criteria were "inflammatory arthritis", "interventions undertaken by nurses" and "relevant outcomes to answer the research questions". Exclusion criteria were in itself contradictory outcomes, insufficient data, consideration if they did not clearly distinguish between nurses and health professionals or focused on chronic other than rheumatic diseases. Systematic reviews were classified as descriptive and excluded. Quality of selected trials was determined according to Oxford-levels of evidence 2009. A total of 48 articles and 10 abstracts were identified fulfilling the eligibility and exclusion criteria. Recommendation 1 has been well established in Europe so far. New evidence strengthens the recommendation 3, and-at least in part—recommendation 6. High evidence strengthens recommendation 4, especially for outpatients with low and stable disease activity. Some new evidence also exists for recommendations 7 and 8. This SLR reveals new evidence for the role of nurses in managing CIA patients since 2010, especially for RA-patients with low disease activity or in remission.
\end{abstract}

Keywords Public health · Organization and administration · Supply and distribution $\cdot$ Nursing $\cdot$ Nurse clinicians

\section{Introduction}

In 2014, the World Forum on Rheumatic and Musculoskeletal Diseases identified "worldwide and regional shortfalls in the provision of rheumatologists", ranging from an estimated 0.5-3.8 rheumatologists per 100,000 inhabitants in Europe, compared to 0.07-3.09 in the Americas and 0.01-0.22 in Asia [1]. Especially in the countries with deficits of qualified rheumatologists, nurses already have an important role in the follow-up and treatment of patients with chronic inflammatory arthritis (CIA), especially cooperate support rheumatologists' work. In order to define recommendations for

Electronic supplementary material The online version of this article (https://doi.org/10.1007/s00296-018-4135-9) contains supplementary material, which is available to authorized users.

Schirmer Michael

michael.schirmer@i-med.ac.at

1 Department of Internal Medicine, Clinic II, Medical University of Innsbruck, Anichstrasse 35, 6020 Innsbruck, Austria the nurses' role, the European League Against Rheumatism (EULAR) searched for evidence supporting standards of care and already in 2011 published European recommendations for the potential role of nurses in the management of patients with chronic inflammatory arthritis [2]. Indeed, the reduced care offered to patients with rheumatoid arthritis (RA) after moving to a nursing home can be considered as an alarming sign for health care planners [3, 4]. EULAR searched for standards of care provided by nurses across the countries for patients with chronic inflammatory arthritis and formulated minimum standards of care [5].

Since then, the EULAR recommendations were well disseminated and positively evaluated both across Europe and the United States (US) [5]. In an online survey, nurses, rheumatologists and patients highly agreed with them but application varied and was lowest in southern, eastern and central Europe. Differences across the countries and the need for further standardisation and research are evident.

The objective of this systematic review was to perform a literature search from 2010 to 2018 on the role of nurses in the management of chronic inflammatory arthritis as a 
follow-up of the 2011 EULAR recommendations using the PRISMA 2009 checklist (supplementary table 1), to evaluate the new trials according to the guidelines of the Oxford Centre for Evidence-based Medicine 2009.

\section{Methods}

A systematic review was performed according to the PRISMA guidelines (supplementary table 1) [6]. The EULAR recommendations [2] were considered as protocol for this work concerning information sources, search terms and eligibility criteria.

\section{Search strategy: information sources and eligibility criteria}

Chronic inflammatory arthritis (CIA) was defined as rheumatoid arthritis (RA), ankylosing spondylitis (AS) and psoriatic arthritis (PsA). The search items are listed in supplementary table 2, mainly including "inflammatory arthritis" and "nurse". MEDLINE was scanned from 01/01/2010 to 01/07/2018, additional search was performed in Cochrane CENTRAL (via OVID SP Search), EMBASE (here via STN), Cumulative Index to Nursing and Allied Health Literature (CINAHL) and Psych Info (via EBSCO host search) available from 01/01/2010 to 18/09/2016.

\section{Article selection}

The eligibility criteria were "inflammatory arthritis", "interventions undertaken by nurses" and "relevant outcomes to answer the research questions" (with details outlined in supplementary table 2). No additional assumptions and simplifications were made. Duplicates were sorted out, including those already considered by the EULAR task force. Articles which did not fulfil the inclusion criteria had contradictory outcomes in itself or insufficient data were excluded. Articles were also considered as insufficient without abstract or study protocols published without data. Articles were not taken into consideration if they did not clearly distinguish between nurses and health professionals or focused on chronic other than rheumatic diseases. Systematic reviews were classified as descriptive and excluded. Prior to exclusion, they were checked for included articles which could be taken into consideration. Only abstracts from the past 2 years were taken into consideration, if evidence was high or concept was interesting for future research.

Both, titles and abstracts of the articles were screened by VL (Vivienne Lion) for fulfilling the eligibility criteria; their relevance was discussed with MS (Michael Schirmer). As the meaning of titles could be misleading, titles and abstracts were screened in one step.

\section{Data collection process}

Data were extracted from reports by VL with subsequent review and discussion with MS. Literature references were collected using Mendeley Desktop (Elsevier Incorporated, New York, New York, USA). Studies were characterised by country of origin, to allow referrals to different health care systems.

\section{Assessment of literature quality and risk of bias}

Quality of additional evidence was assessed according to the Oxford Centre for Evidence-based Medicine 2009. Identified abstracts which were not excluded by the eligibility criteria were not considered for any evidence category, as data were not fully available. Risk of bias was considered in trials on pharmaceuticals and medical devices, which were supported by the producing companies. There was no need to assess statistical methods as part of the meta-analysis. No risk of bias was searched for, like financial or personal bias.

\section{Results}

\section{Study selection and characteristics}

A total of 2515 articles were screened, and finally 48 articles and 10 abstracts assessed as eligible and included in this review (Fig. 1): 1 meta-analysis, 17 randomised controlled trials (RCTs, summarised in Table 1), 6 quasiexperimental studies, 5 observational studies, 3 crosssectional studies and 16 qualitative studies. Thirty-one articles exclusively dealt with the management of RA and 12 dealt with CIAs including RA. Three studies focused on rheumatic diseases in general and two on inflammatory rheumatic diseases. None of the identified articles solely considered PsA or AS. Specialised nursing personnel participated in 36 identified studies. Categories of additional evidence are presented in Table 2. Articles with studies of low evidence and abstracts were cited in this review only when higher evidence was not available. After consideration of a possible risk of bias in trials on pharmaceuticals and medical devices, which were supported by the producing companies, none of the studies was included in this SLR (supplementary table 1). No personal risk of bias was identified across the studies. 
Fig. 1 Flowchart for the results of the systematic literature review (MEDLINE (OVID) 01/01/10-01/07/18, additional searches available from 01/01/10 to $18 / 09 / 16$ )

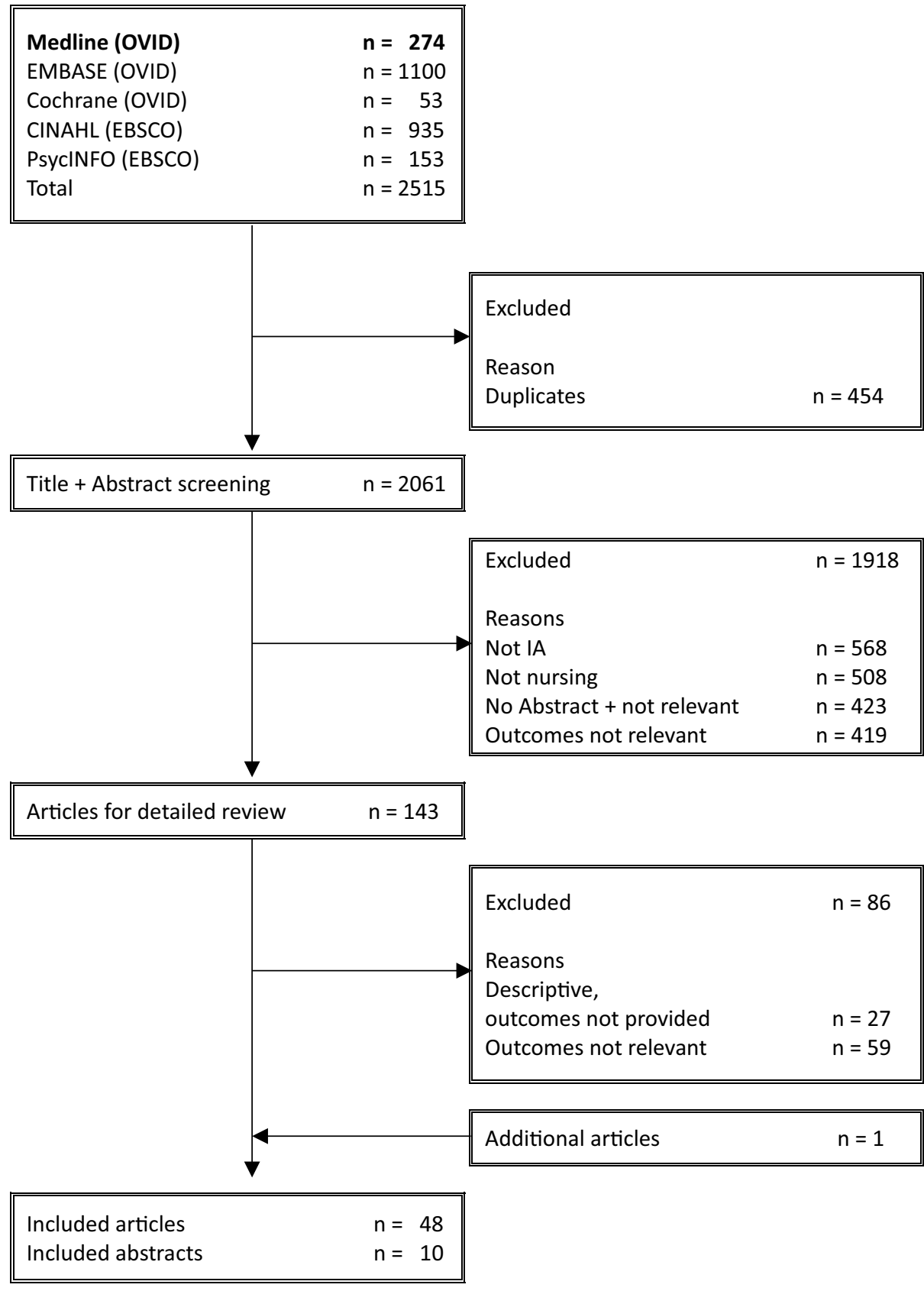

\section{Synthesis of results}

Recommendation 1 "Patients should have access to a nurse for education to improve knowledge of CIA and its management throughout the course of their disease"

Four RCTs reported benefits for patients: several trials aimed at improving not only patients' knowledge [7] but also patients' self-care ability [8] and self-efficacy [9, 10], global well-being [7, 9], empowerment [8], beliefs and behaviours to manage chronic illness [9]. In addition, education resulted in trained patients showing increased physical activity [10], able to reliably determine their disease activity [11] and being more probable to quit smoking [12]. In Europe, such education provided by non-physician health professionals is well established in 24 of 27 countries [13].

Recommendation 2 "Patients should have access to nurse consultations in order to experience improved communication, continuity and satisfaction with care"

A recent meta-analysis did not find a difference between nurse-led and physician-led follow-up after 1 and 2 years, even with low-evidence favour of nurse-led follow-up in patients with low disease-activity [14]. Direct comparisons in RCTs resulted in divergent priorities for nurses or physicians' consultations [15-17]. After 1 year, satisfaction was 


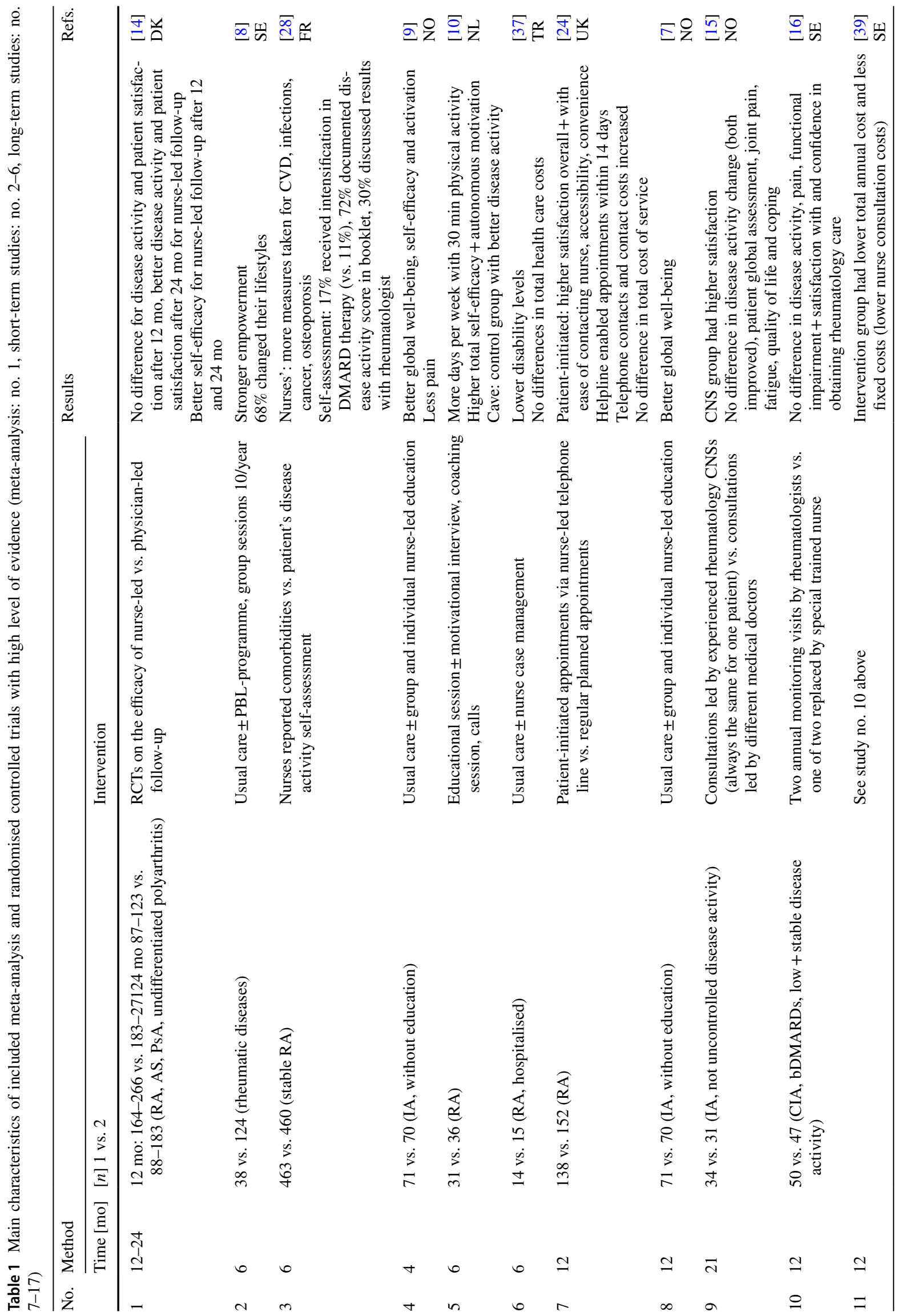




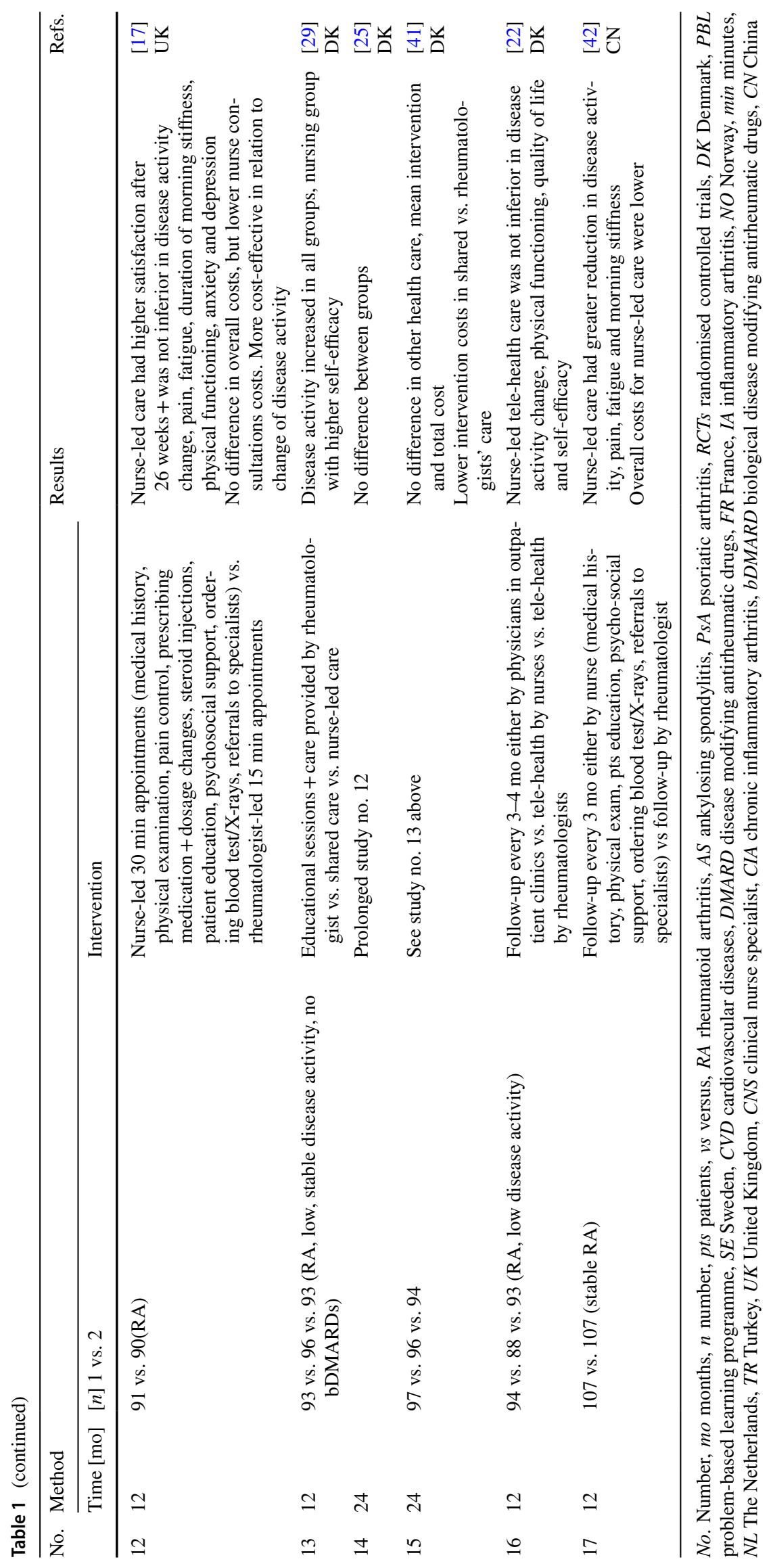


Table 2 Additional evidence of 2010-2018 literature for recommendations of rheumatology nursing management in CIA according to Oxford levels of evidence 2009

EULAR recommendations 2011

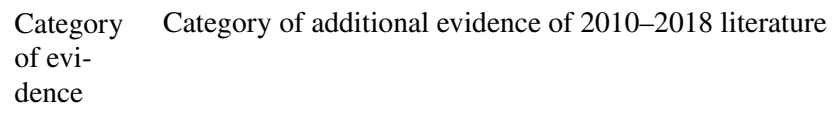

dence

1 Patients should have access to a nurse for education to improve knowledge of CIA and its management throughout the course of their disease

2 Patients should have access to nurse consultations in order to experience improved communication, continuity and satisfaction with care

3 Patients should have access to nurse-led telephone services to enhance continuity of care and to provide ongoing support

4 Nurses should participate in comprehensive disease management to control disease activity, to reduce symptoms and to improve patientpreferred outcome

5 Nurses should identify, assess and address psychosocial issues to minimise the chance of patients' anxiety and depression

6 Nurses should promote self-management skills in order that patients might achieve a greater sense of control, self-efficacy and empowerment

7 Nurses should provide care that is based on protocols and guidelines according to national and local contexts

8 Nurses should have access to and undertake continuous education in 3 order to improve and maintain knowledge and skills

9 Nurses should be encouraged to undertake extended roles after spe- 3 cialised training and according to national regulations

10 Nurses should carry out interventions and monitoring as part of com- 1B prehensive disease management in order to achieve cost savings

\begin{tabular}{|c|c|}
\hline 1B & $1 \mathrm{~B}$ \\
\hline $1 \mathrm{~B}$ & $\begin{array}{l}\text { 1A for satisfaction with care } \\
4 \text { for improved communication and continuity }\end{array}$ \\
\hline 3 & $1 \mathrm{~B}$ \\
\hline $1 \mathrm{~A}$ & $1 \mathrm{~A}$ \\
\hline $1 \mathrm{~B}$ & $1 \mathrm{~B}$ \\
\hline 3 & $\begin{array}{l}\text { 1A for self-efficacy } \\
\text { 2B for empowerment Sense of control not studied }\end{array}$ \\
\hline 3 & $2 \mathrm{~B}$ \\
\hline 3 & $2 \mathrm{~B}$ \\
\hline 3 & 3 \\
\hline $1 \mathrm{~B}$ & $1 \mathrm{~B}$ \\
\hline
\end{tabular}

equally estimated by CIA-patients with low disease activity under treatment with biologic agents, if physician-led care was replaced by a rheumatology nurse every second visit and nurses' service of $30 \mathrm{~min}$ was preferred to physicians' service of $15 \mathrm{~min}$ after 26 weeks but not after 52 weeks [17]. Qualitative studies confirmed the recommendation concerning improved communication [18-20] and continuity [19, 21] as experienced by the patients.

Recommendation 3 "Patients should have access to nurseled telephone services to enhance continuity of care and to provide ongoing support"

According to a recent RCT, an outcome-based tele-health follow-up for tight control of RA patients with low disease activity or remission can achieve similar disease control as conventional outpatient follow-up by rheumatologists [22]. Already earlier, one RCT and one quasi-experimental study showed that telephone services increase patients'; empowerment [23] and satisfaction, [23, 24] enhance their motivation [19] and ensure safety under treatment. Besides, nurse-led telephone services have been established to provide additional care and as such are incorporated in different interventional services at least in 15 of 27 European countries [13]. Telephone services may also make access to care easier, but may also be initiated by the nurse.
Recommendation 4 "Nurses should participate in comprehensive disease management to control disease activity, to reduce symptoms and to improve patient-preferred outcomes"

Many studies including an RCT further investigated clinical outcomes using disease activity scores [7, 9, 15-17, 25-29]. Overall outcome of nurse-led care was not inferior to rheumatologists' care as measured by disease activity in patients with low disease activity or remission, and maybe replaced even by outcome-based tele-health follow-up by nurses [22].

Recommendation 5 "Nurses should identify, assess and address psychosocial issues to minimise the chance of patients' anxiety and depression"

In two RCTs, nurses' interventions did not minimise patients' anxiety and depression [7, 9]. Nurse-led education may improve global well-being but not necessarily patients' psychosocial health [7, 9]. According to a survey, $74 \%$ of CIA patients preferred psychological support provided by a nurse compared to $55 \%$ by a physician [30].

Recommendation 6 "Nurses should promote selfmanagement skills in order that patients might achieve a greater sense of control, self-efficacy and empowerment" 
New RCTs studied different self-management skills (e.g. promoting education and physical exercise) and further confirmed that nurse-led interventions lead to higher selfefficacy $[9,10,29]$ and more empowerment [23], especially among RA-patients, as confirmed for self-efficacy by a recent meta-analysis [14]. Sense of control was not studied.

Recommendation 7 "Nurses should provide care that is based on protocols and guidelines according to national and local contexts"

Guidelines and protocols most often referred to medical treatment and guide in monitoring visits during treatment with biological agents. Nurse practitioners benefited very strongly from an educational programme to further improve the management of RA [31, 32].

Recommendation 8 "Nurses should have access to and undertake continuous education to improve and maintain knowledge and skills"

Indeed, after various training programmes, nurses took over new roles [33-37] or improved their performance in the management of patients [31, 32, 38]. They performed joint examinations [33, 34] and examined gait, arms, legs and spine to distinguish between RA or non-RA [35]. Overall their work profile changed, as they gained more independence, took a more specific medical history, supported studies, provided information on infusions and administered those [36].

Recommendation 9 "Nurses should be encouraged to undertake extended roles after specialised training and according to national regulations"

Many RCTs with participation of specialised personnel were identified. Extended roles of nurse care include consultant role, advanced clinical tasks, administration of intraarticular injections and managing patient advice lines [13]. Legal constraints may limit the wide-spread performance of these roles. For the professionals themselves, specialised training led to higher work satisfaction, more independent work and implementation of new tasks [36], and increased self-confidence, knowledge and career opportunities for the nurses [13].

Recommendation 10 "Nurses should carry out interventions and monitoring as part of comprehensive disease management in order to achieve cost savings"

Cost reductions were reported together with stable outcome parameters in monitoring CIA-outpatients with stable and low disease activity under treatment with biological agents, with a nurse taking over every second visit of the rheumatologist [39]. Authors from different European countries performed economic analyses of various forms of nurse-led care, and the majority of economic analyses investigated an established nurse-led model of care [17, 26, 39, 40] provided by specialised nurses [17, 39-41]. Although nurse consultation costs were lower than physicians' costs, there was only few evidence that nurse-led care decreases total costs compared to physician-led care [39]. When including loss of productivity into total costs, costs of nurseled community care may be even higher than hospital care [40]. Cost-effectiveness studies have not been considered for this SLR, if disease activity outcome parameters were not comparable [42].

\section{Discussion}

Taken together, important new evidence for the role of nurses in the management of CIA came up during the past years (outlined in Table 2), especially for recommendations 3 and 6 . There was no contradictory evidence to any of the recommendations. Therefore, as additional provision of care to patients with rheumatic diseases will be needed in the future, nurses will be able to support rheumatologists, especially in CIA patients with stable disease and low disease activity.

Based on current evidence, the professional role of nurses will certainly change, especially in out-patient clinics. Depending on the recommendation addressed, however, the quality of new evidence widely differs. For recommendations 1 and 3 it appears that more evidence from randomised clinical trials will hardly change the clinical practice in the future, as in many European rheumatologic services patients have already access to nurses [13] and nurse-led telephone services not only in trials but also in routine clinical settings [13, 19, 43-46]. In fact, outcome-based tele-health services by nurses may even replace nurse-led visits in RA patients with low disease activity or in remission [22]. As a next step, evidence for new technical tools is growing as for mail services [24, 44, 47, 48].

The main tasks of nurses in rheumatic services, to perform a comprehensive disease management to control disease activity, to reduce symptoms and to improve patientpreferred outcomes have also been further supported by new evidence - and there is certainly more space for other responsibilities of nurses in the future, especially in the disease management of outpatients with low and stable disease activity [15, 16, 25, 29, 40]. For these tasks nurses' support by other health care professionals may be helpful, as has been shown for example to increase physical activity [10] and to quit smoking [12].

Besides, evidence increases for nurse-led education, especially for CIA-patients with stable disease which led to improved global well-being [7, 9], self-efficacy [9, 10], self-assessment of disease activity [11], empowerment [8], activation [9] and knowledge [7]. Besides, nurses can contribute to self-injection training of medical treatment [49, 50], train disease-activity self-assessment [28], follow-up care after a self-care promoting programme [29] and selfregulation sessions together with follow-up phone calls [10]. 
Initiatives are ongoing to improve the quality of nurse-led education [51]. The use of an educational needs assessment tool (ENAT) like for RA in the UK [51] allows patients to indicate their educational needs at each rheumatologic visit to further increase their self-efficacy by focusing on their individual needs [52]. Translations of ENAT for RA-patients are available for nine European languages so far [52].

For the future, educational efforts for the patients will have to concentrate on long-term repetitive interactions to ensure a minimum level of patients' knowledge during the course of the disease, sometimes even leading to change of lifestyle behaviour $[8,10]$. RA- and PsA-patients trained by a rheumatologist and a health psychologist may become more independent in interpreting blood results and checking for side effects of MTX therapy themselves, resulting in up to $55 \%$ fewer visits to the clinical nurse specialist, $7 \%$ fewer visits to the rheumatologist and $39 \%$ fewer visits to the general practitioner, when nurses only provide a telephone helpline as usual care [43]. This may reduce unnecessary appointments at the rheumatology clinics and even total costs of rheumatologic care in the future-given a comparable or even better outcome. Calculations of costs with adjustments for health care quality solely based on questionnaires, however, appear critical and available data are not convincing. As a matter of fact, specific training programmes for nurses are necessary before they can take over new roles [33-37, 53] or improve their performance in the management of patients $[32,38,54]$. Guidelines and protocols have to be developed and further disseminated among professionals involved in care [54]. Besides, more studies on nurses' care have to be performed for SpA and PsA, as the level of evidence is higher for nurses caring for RA patients than for SpA and PsA.

The most important strength of this review is that it was performed according to the PRISMA guidelines [6]. Besides, a detailed table was set up to summarize the characteristics of the included studies (Table 1).

A limitation of this review is that most studies on nurses' care have been performed for RA patients, and the level of evidence is higher for nurses caring for RA patients than for SpA and PsA. Also, most studies focus on patients in stable and low disease activity.

Based on this SLR, future trials are needed, especially for nurse-led services to patients with diseases other than CIA, to define extended roles of nurse-led services like supporting triage efforts, implementing treat-to-target guidelines and Improving effective utilisation of care by multidisciplinary teams.

In conclusion, there is increasing evidence for the role of nurses in the management of patients with chronic inflammatory arthritis. Some recommendations are already practiced in routine clinical work, whereas RCTs are still needed for others.
Acknowledgements Open access funding provided by University of Innsbruck and Medical University of Innsbruck.

\section{Compliance with ethical standards}

Conflict of interest The authors declare that they have no conflict of interest.

Open Access This article is distributed under the terms of the Creative Commons Attribution 4.0 International License (http://creativeco mmons.org/licenses/by/4.0/), which permits unrestricted use, distribution, and reproduction in any medium, provided you give appropriate credit to the original author(s) and the source, provide a link to the Creative Commons license, and indicate if changes were made.

\section{References}

1. Al Maini M, Adelowo F, Al Saleh J et al (2015) The global challenges and opportunities in the practice of rheumatology: white paper by the World Forum on Rheumatic and Musculoskeletal Diseases. Clin Rheumatol 34:819-829. https://doi.org/10.1007/ s10067-014-2841-6

2. van Eijk-Hustings Y, van Tubergen A, Boström C et al (2012) EULAR recommendations for the role of the nurse in the management of chronic inflammatory arthritis. Ann Rheum Dis 71:13-19. https://doi.org/10.1136/annrheumdis-2011-200185

3. Luque Ramos A, Albrecht K, Zink A, Hoffmann F (2017) Rheumatologic care of nursing home residents with rheumatoid arthritis: a comparison of the year before and after nursing home admission. Rheumatol Int. https://doi.org/10.1007/s0029 6-017-3791-5

4. Schirmer M, Lion V (2017) Decline of rheumatologic care of nursing home residents with rheumatoid arthritis. Rheumatol Int 37:1947-1948. https://doi.org/10.1007/s00296-017-3810-6

5. van Eijk-Hustings Y, Ndosi M, Buss B et al (2014) Dissemination and evaluation of the European League Against Rheumatism recommendations for the role of the nurse in the management of chronic inflammatory arthritis: results of a multinational survey among nurses, rheumatologists and patients. Rheumatology 53:1491-1496. https://doi.org/10.1093/rheumatology/keu134

6. Moher D, Liberati A, Tetzlaff J, Altman DG (2009) Preferred reporting items for systematic reviews and meta-analyses: the PRISMA statement. BMJ 339:b2535-b2535. https://doi. org/10.1136/bmj.b2535

7. Grønning K, Rannestad T, Skomsvoll JF et al (2014) Long-term effects of a nurse-led group and individual patient education programme for patients with chronic inflammatory polyarthritis-a randomised controlled trial. J Clin Nurs 23:1005-1017. https:// doi.org/10.1111/jocn.12353

8. Arvidsson S, Bergman S, Arvidsson B et al (2013) Effects of a self-care promoting problem-based learning programme in people with rheumatic diseases: a randomized controlled study. J Adv Nurs 69:1500-1514. https://doi.org/10.1111/jan.12008

9. Grønning K, Skomsvoll JF, Rannestad T, Steinsbekk A (2012) The effect of an educational programme consisting of group and individual arthritis education for patients with polyarthritis-a randomised controlled trial. Patient Educ Couns 88:113-120. https://doi.org/10.1016/j.pec.2011.12.011

10. Knittle K, De Gucht V, Hurkmans E et al (2015) Targeting motivation and self-regulation to increase physical activity among patients with rheumatoid arthritis: a randomised controlled trial. 
Clin Rheumatol 34:231-238. https://doi.org/10.1007/s1006 7-013-2425-x

11. Cheung PP, Ruyssen-Witrand A, Gossec L et al (2010) Reliability of patient self-evaluation of swollen and tender joints in rheumatoid arthritis: a comparison study with ultrasonography, physician, and nurse assessments. Arthritis Care Res (Hoboken) 62:1112-1119. https://doi.org/10.1002/acr.20178

12. Naranjo A, Bilbao A, Erausquin C et al (2014) Results of a specific smoking cessation program for patients with arthritis in a rheumatology clinic. Rheumatol Int 34:93-99. https://doi. org/10.1007/s00296-013-2851-8

13. Stamm T, Hill J (2011) Extended roles of non-physician health professionals and innovative models of care within Europe: results from a web-based survey. Musculoskelet Care 9:93-101

14. de Thurah A, Esbensen BA, Roelsgaard IK et al (2017) Efficacy of embedded nurse-led versus conventional physician-led follow-up in rheumatoid arthritis: a systematic review and meta-analysis. RMD Open 3:e000481. https://doi.org/10.1136/rmdopen-2017000481

15. Koksvik HS, Hagen KB, Rødevand E et al (2013) Patient satisfaction with nursing consultations in a rheumatology outpatient clinic: a 21-month randomised controlled trial in patients with inflammatory arthritides. Ann Rheum Dis 72:836-843. https:// doi.org/10.1136/annrheumdis-2012-202296

16. Larsson I, Fridlund B, Arvidsson B et al (2014) Randomized controlled trial of a nurse-led rheumatology clinic for monitoring biological therapy. J Adv Nurs 70:164-175. https://doi.org/10.1111/ jan. 12183

17. Ndosi M, Lewis M, Hale C et al (2014) The outcome and costeffectiveness of nurse-led care in people with rheumatoid arthritis: a multicentre randomised controlled trial. Ann Rheum Dis 73:1975-1982. https://doi.org/10.1136/annrheumdis-2013-20340 3

18. Larsson I, Bergman S, Fridlund B, Arvidsson B (2012) Patients' experiences of a nurse-led rheumatology clinic in Sweden: a qualitative study. Nurs Health Sci 14:501-507. https://doi.org/10 $.1111 / j .1442-2018.2012 .00723 . x$

19. Bala S-V, Samuelson K, Hagell P et al (2012) The experience of care at nurse-led rheumatology clinics. Musculoskelet Care 10:202-211. https://doi.org/10.1002/msc.1021

20. Vinall-Collier K, Madill A, Firth J (2016) A multi-centre study of interactional style in nurse specialist- and physician-led rheumatology clinics in the UK. Int J Nurs Stud 59:41-50. https://doi. org/10.1016/j.ijnurstu.2016.02.009

21. Primdahl J, Wagner L, Hørslev-Petersen K (2011) Being an outpatient with rheumatoid arthritis - a focus group study on patients' self-efficacy and experiences from participation in a short course and one of three different outpatient settings. Scand J Caring Sci 25:394-403. https://doi.org/10.1111/j.1471-6712.2010.00854.x

22. de Thurah A, Stengaard-Pedersen K, Axelsen M et al (2018) Tele-health followup strategy for tight control of disease activity in rheumatoid arthritis: results of a randomized controlled trial. Arthritis Care Res (Hoboken) 70:353-360. https://doi. org/10.1002/acr.23280

23. Johansson K, Katajisto J, Salanterä S (2010) Pre-admission education in surgical rheumatology nursing: towards greater patient empowerment. J Clin Nurs 19:2980-2988. https://doi.org/10.111 1/j.1365-2702.2010.03347.x

24. Goodwin VA, Paudyal P, Perry MG et al (2016) Implementing a patient-initiated review system for people with rheumatoid arthritis: a prospective, comparative service evaluation. J Eval Clin Pract 22:439-445. https://doi.org/10.1111/jep.12505

25. Primdahl J, Petersen R, Horslev-Petersen K, Horn H (2013) Clinical and radiological outcome in outpatients with rheumatoid arthritis followed by medical, nursing or shared care-a 2 years randomised controlled trial. Ann Rheum Dis 71:92
26. Muñoz-Fernández S, Aguilar MD, Rodríguez A et al (2016) Evaluation of the impact of nursing clinics in the rheumatology services. Rheumatol Int 36:1309-1317. https://doi.org/10.1007/ s00296-016-3518-z

27. Solomon DH, Fraenkel L, Lu B et al (2015) Comparison of care provided in practices with nurse practitioners and physician assistants versus subspecialist physicians only: a cohort study of rheumatoid arthritis. Arthritis Care Res (Hoboken) 67:1664-1670. https://doi.org/10.1002/acr.22643

28. Dougados M, Soubrier M, Perrodeau E et al (2015) Impact of a nurse-led programme on comorbidity management and impact of a patient self-assessment of disease activity on the management of rheumatoid arthritis: results of a prospective, multicentre, randomised, controlled trial (COMEDRA). Ann Rheum Dis 74:1725-1733. https://doi.org/10.1136/annrheumdi s-2013-204733

29. Primdahl J, Wagner L, Holst R, Hørslev-Petersen K (2012) The impact on self-efficacy of different types of follow-up care and disease status in patients with rheumatoid arthritis-a randomized trial. Patient Educ Couns 88:121-128. https://doi. org/10.1016/j.pec.2012.01.012

30. Dures E, Almeida C, Caesley J et al (2016) Patient preferences for psychological support in inflammatory arthritis: a multicentre survey. Ann Rheum Dis 75:142-147. https://doi. org/10.1136/annrheumdis-2014-205636

31. Lineker SC, Bell MJ, Badley EM (2011) Evaluation of an inter-professional educational intervention to improve the use of arthritis best practices in primary care. J Rheumatol 38:931937. https://doi.org/10.3899/jrheum.101007

32. Lineker SC, Husted JA, Brown KS (2012) Influence of discipline of provider and model of care on an arthritis educational intervention in primary care. Arthritis Care Res (Hoboken) 64:424-433. https://doi.org/10.1002/acr.20694

33. Cheung PP, Dougados M, Andre V et al (2014) The learning curve of nurses for the assessment of swollen and tender joints in rheumatoid arthritis. Jt Bone Spine 81:154-159. https://doi. org/10.1016/j.jbspin.2013.06.006

34. Amity CL, Schlenk EA, Gold KN et al (2016) Agreement of physicians and nurses performing tender and swollen joint counts in rheumatoid arthritis. JCR J Clin Rheumatol 22:30-34. https://doi.org/10.1097/RHU.0000000000000324

35. Beattie KA, MacIntyre NJ, Cividino A (2012) Screening for signs and symptoms of rheumatoid arthritis by family physicians and nurse practitioners using the gait, arms, legs, and spine musculoskeletal examination. Arthritis Care Res (Hoboken) 64:1923-1927. https://doi.org/10.1002/acr.21740

36. Schuch F, Kern P, Kreher G et al (2011) Das Curriculum "Rheumatologische Fachassistenz DGRh-BDRh" als Weg der Professionalisierung medizinischer Assistenzberufe. Z Rheumatol 70:670-677. https://doi.org/10.1007/s00393-011-0840-4

37. Tokem Y, Argon G, Keser G (2011) Case management in care of Turkish rheumatoid arthritis patients. Rehabil Nurs 36:205-213. https://doi.org/10.1002/j.2048-7940.2011.tb00197.x

38. Lesuis N, Verhoef LM, Nieboer LM et al (2017) Implementation of protocolized tight control and biological dose optimization in daily clinical practice: results of a pilot study. Scand J Rheumatol 46:152-155. https://doi.org/10.1080/03009742.2016.11944 57

39. Larsson I, Fridlund B, Arvidsson B et al (2015) A nurse-led rheumatology clinic versus rheumatologist-led clinic in monitoring of patients with chronic inflammatory arthritis undergoing biological therapy: a cost comparison study in a randomised controlled trial. BMC Musculoskelet Disord 16:354. https://doi.org/10.1186/ s12891-015-0817-6

40. Watts RA, Mooney J, Barton G et al (2015) The outcome and costeffectiveness of nurse-led care in the community for people with 
rheumatoid arthritis: a non-randomised pragmatic study. BMJ Open 5:e007696. https://doi.org/10.1136/bmjopen-2015-007696

41. Sørensen J, Primdahl J, Horn H, Hørslev-Petersen K (2015) Shared care or nurse consultations as an alternative to rheumatologist follow-up for rheumatoid arthritis (RA) outpatients with stable low disease-activity RA: cost-effectiveness based on a 2-year randomized trial. Scand J Rheumatol 44:13-21. https:// doi.org/10.3109/03009742.2014.928945

42. Junru W, Xiulan Z, Ling C, Huina L (2017) Clinical effectiveness and cost-effectiveness of nurse-led care in Chinese patients with rheumatoid arthritis: A randomized trial comparing with rheumatologist-led care. Int J Nurs Pract 24:e12605. https://doi. org/10.1111/ijn.12605

43. McBain H, Shipley M, Olaleye A et al (2016) A patient-initiated DMARD self-monitoring service for people with rheumatoid or psoriatic arthritis on methotrexate: a randomised controlled trial. Ann Rheum Dis 75:1343-1349. https://doi.org/10.1136/annrh eumdis-2015-207768

44. Garcia Diaz S, Samper E, Roig Vilaseca D et al (2015) FRI0634HPR non attending rheumatology nursing consultations, high resolution of urgent demand [abstract]. Ann Rheum Dis 74:13301331. https://doi.org/10.1136/annrheumdis-2015-eular.4612

45. Nájera Herranz C, Ivorra Cortés J, Chalmeta Verdejo I et al (2015) FRI0621-HPR Usefulness of the telephone nursing consultation for the monitoring of patients treated with subcutaneous therapy. Two years of results [abstract]. Ann Rheum Dis 74:1326. https:// doi.org/10.1136/annrheumdis-2015-eular.5979

46. Bellerby T, Stevens D, Arnold T et al (2016) AB1085-HPR audit of nurse-led DGH rheumatology advice line [abstract]. Ann Rheum Dis 75:1302-1303. https://doi.org/10.1136/annrheumdi s-2016-eular.5630

47. Hoving J, Zoer I, van der Meer M et al (2014) E-health to improve work functioning in employees with rheumatoid arthritis in rheumatology practice: a feasibility study. Scand J Rheumatol 43:481-487. https://doi.org/10.3109/03009742.2014.914568

48. Cano-Garcia L, Manrique-Arija S, Ureña I et al (2016) AB1078HPR Telephone follow-up, standardized to the initiation of biologic therapy of patients with rheumatoid arthritis (RA) in a specific unit of biologic therapy. Pilot study [abstract]. Ann Rheum Dis 75:1300. https://doi.org/10.1136/annrheumdis-2016-eular .1432

49. Fitzgerald P, Hale C (2014) Moving the injectable methotrexate service closer to home: a practice development initiative. Musculoskeletal Care 12:182-189. https://doi.org/10.1002/msc.1070

50. Fall E, Chakroun N, Dalle N, Izaute M (2013) Is patient education helpful in providing care for patients with rheumatoid arthritis? A qualitative study involving French nurses. Nurs Health Sci 15:346-352. https://doi.org/10.1111/nhs. 12042

51. Ndosi M, Johnson D, Young T et al (2016) Effects of needs-based patient education on self-efficacy and health outcomes in people with rheumatoid arthritis: a multicentre, single blind, randomised controlled trial. Ann Rheum Dis 75:1126-1132. https://doi. org/10.1136/annrheumdis-2014-207171

52. Hardware B, Johnson D, Hale C et al (2015) Patients and nursing staff views of using the education needs assessment tool in rheumatology clinics: a qualitative study. J Clin Nurs 24:1048-1058. https://doi.org/10.1111/jocn.12733

53. Salomon M, Litinsky I, Almog R, Elkayam O (2015) FRI0630HPR assessment of disease activity in rheumatic patients by rheumatology nurses - feasibility study of validity and practicability [abstract]. Ann Rheum Dis 74:1329. https://doi.org/10.1136/annrh eumdis-2015-eular.2500

54. Lineker S, Bell M, Badley E (2011) Evaluation of an inter-professional educational intervention to improve the use of arthritis best practices in primary care. J Rheumatol 38:931-937. https:// doi.org/10.3899/jrheum.101007 